\title{
The Doubling of the Degrees of Freedom in Quantum Dissipative Systems, and the Semantic Information Notion and Measure in Biosemiotics ${ }^{+}$
}

\author{
Gianfranco Basti ${ }^{1, *}$, Antonio Capolupo ${ }^{2,3}$ and GiuseppeVitiello ${ }^{2}$ \\ 1 Faculty of Philosophy, Pontifical Lateran University, 00120 Vatican City, Italy \\ 2 Department of Physics “E:R. Caianiello”, University of Salerno, Via Giovanni Paolo II, 132, \\ 84084 Fisciano (SA), Italy; capolupo@sa.infn.it (A.C.); vitiello@sa.infn.it (G.V.) \\ 3 Istituto Nazionale di Fisica Nucleare (INFN), Gruppo Collegato di Salerno, Via Giovanni Paolo II, 132, \\ 84084 Fisciano (SA), Italy \\ * Correspondence: basti@pul.va; Tel.: +39-339-576-0314 \\ + Conference Theoretical Information Studies (TIS), Berkeley, CA, USA, 2-6 June 2019. \\ Published: 11 May 2020
}

Keywords: semantic information; non-equilibrium thermodynamics; quantum field theory

\section{The Semantic Information Issue and Non-Equilibrium Thermodynamics}

In the recent history of the effort for defining a suitable notion and measure of semantic information, starting points are: the "syntactic" notion and measure of information $H$ of Claude Shannon in the mathematical theory of communications (MTC) [1]; and the "semantic" notion and measure of information content or informativeness of Rudolph Carnap and Yehoshua Bar-Hillel [2], based on Carnap's theory of intensional modal logic [3].

The relationship between $H$ and the notion and measure of entropy $S$ in Gibbs' statistical mechanics (SM) and then in Boltzmann's thermodynamics is a classical one, because they share the same mathematical form.

We emphasize that Shannon's information measure is the information associated to an event in quantum mechanics (QM) [4]. Indeed, in the classical limit, i.e., whenever the classical notion of probability applies, $S$ is equivalent to the QM definition of entropy given by John Von Neumann. Indeed, he prompted Claude Shannon to call "entropy" the statistical measure of information $H$. As emphasized by Shannon himself [1] (p. 3), the "syntactic" and not "semantic" character of $H$ and its connection with entropy $S$ in $\mathrm{SM}$ and QM, is that the "informativeness" is associated with the "uncertainty removal". This is determined with the occurrence of a symbol in MTC and/or of an event in SM or in QM [4].

Another point is the classical semantic information (CSI) theory in the logic of Carnap and Bar-Hillel [2]. There the intension of a given sentence corresponds to the set of "possible worlds" (or "contexts", defined according to Carnap's intensional modal logic [3]) that make the sentence true. This theory leads to the famous Carnap and Bar-Hillel paradox, separating the semantic information content of a given declarative sentence from the notion of logical truth. In fact, because a logical truth is true in all possible worlds, $w \in W$, i.e., it does not exclude any world, it is empty of any semantic content (effectively, it is a tautology). Therefore, the information content of a sentence $s$, Cont $(s)$, is defined in CSI as the set of all sentences (possible worlds) belonging to the same universe $W$ of the theory excluded by $s$.

The paradoxical conclusion is that the maximum semantic content is always a false (i.e., a contradictory) sentence, because it excludes any possible world. In other words, "a self-contradictory sentence asserts too much: it is too informative for being true" ([2], p. 229). 
To correct this separation between truthfulness and informativeness in CSI, by vindicating that a consistent theory of semantic information must concern "well-formed, meaningful, and truthful data", Luciano Floridi proposed his theory of strong semantic information (TSSI) [5]. This measure is based on a "contingency requirement of informativeness", and then on the notion of contingent truth in modal logic (= true for some possible worlds $\left(w_{i} \in W\right)$ ), so that, "a declarative sentence $s$ is informative if $s$ individuates at least some (possibly one: the actual world $w_{0}$ ) but not all $w_{i}$ from $W$ [6]. Now, the probabilistic measure of informativeness $\sigma$ associated with a sentence $s$ proposed by Floridi ([5], p. 55ff.) is a measure of some sort of "distance" $\theta$ of $\sigma$ from the actual world $w_{0}$, so that it is possible to calculate the degree of informativeness $\iota(\sigma)=1-\theta(\sigma)^{2}$, where the maximum of informativeness is of course when $\theta(\sigma)=0$. In this way, both for the "always true" proposition ( $s \mathrm{~V}$ $\neg s)$ or a tautology of CSI, for which $\theta(\sigma)=1$, and for the "always false" proposition of CSI $(s \wedge \neg s)$, for which $\theta(\sigma)=-1$, the degree of informativeness is null, i.e., $\iota(\sigma)=0$, while it is maximum, i.e., $\iota(\sigma)=1$, only for the "contingently true" propositions where $\theta(\sigma)=0$, because it refers to the actual world $w_{0}$.

The problem of the TSSI measure of informativeness is twofold.

On the one hand, it supposes the existence of negative probabilities (between -1 and 0 ) or "pseudo-probabilities", violating the first axiom of the three ones of Andrey N. Kolmogorov's axiomatic theory of probabilities ${ }^{1}$ [7].

On the other hand, TSSI effectively proposes a theory of semantic information independent of the entropy measure in physics and of the information-theoretic approach to universal computations. This second limitation of TSSI is emphasized by Pieter Adriaans [8], who suggests that more interesting would be the development of a theory of "non-equilibrium entropy" ([8], p. 45). Namely, the study of the entropy (the information, and then the universal computations) associated with the thermodynamics of dissipative systems moving through different phases and staying persistently in far-from-equilibrium conditions $[9,10]$.

Antonio Damasio connected the intentional aboutness, or reference-to-object typical of the phenomenological approach, to meaning in psychology [11], primarily to biology, and more specifically to the fundamental notion of homeostasis [12], which expresses much more than the simple "equilibrium" in thermodynamics. He recalls very appropriately that equilibrium in biology is a synonym of "death" and of the simple linear "energy balance". In fact, homeostasis refers in biology to the non-linear phenomena of "self-regulation", ranging from the molecular, to the cellular, to the tissue levels, and so on, till it involves the whole organism and its environment (see the I part of [12], pp. 11-69 for a review of this notion). For instance, the complex self-regulation processes collected under the term epigenetics, studying "heritable changes caused by the activation and deactivation of genes without any change in the underlying DNA sequence of the organism" [13], evidently depends on the interaction between DNA/RNA sequences and the inner/outer chemical environment of cells. This confirms that whichever organism is a dissipative system, not only for its metabolism, but in its most basic component, the genome. This makes the relationship between dissipative systems and semantic information even more interesting, as well as for a proper understanding of the notion of "genetic code", or more properly of "epigenetic code", or "organic code" in biosemiotics $[14,15]$.

\section{Dissipative Systems, the Doubling of the Degrees of Freedom, and the Semantic Information Measure in QFT}

Let $(\Omega, \mathcal{F}, P)$ be a "measure space" with $P$ being a probability of some event $E$ denoted as $P(E)$ and $P(\Omega)=$ 1. Then, $(\Omega, \mathcal{F}, P)$ is a "probability space", with sample space $\Omega$, event space $\mathcal{F}$, and probability measure $P$. (1) First axiom: "The probability of an event is a non-negative real number: $P(E) \in \mathbb{R}, P(E) \geq 0 ; \forall E \in \mathcal{F}$. (2) Second axiom: "The probability that at least one of the elementary events in the whole sample space will occur is 1 ('unit measure' assumption, or 'unitarity' in physics)": $P(\Omega)=1$. (3) Third axiom: "Any countable sequence of disjoint (mutually exclusive) events $E_{1}, E_{2}, \ldots$ satisfies $P\left(\cup_{i=1}^{\infty} E_{1}\right)=\sum_{i=1}^{\infty} P\left(E_{i}\right)$ (= ' $\sigma$-additivity' assumption). We note that quasi-probability distributions relax this axiom. In quantum physics they are typical of coherent states (phases): $P\left(\left|\alpha_{0}\right\rangle\right)$, where $P$ is a delta function: $P\left(\alpha, \alpha^{*}\right)=\delta^{2}\left(\alpha-\alpha_{0}\right)$, e.g., in the Wigner function in QM and QFT systems. 
Since the publication of its preprint at the end of 2019, a paper just published on Nature Communications [16] excited the debate among scientists because it demonstrated, in an experiment of matter-wave interferometry, quantum self-interferences, similar to the most famous "double-slit" experiment in $\mathrm{QM}$, of a natural polypeptide, the gramicidin A1, a linear antibiotic polypeptide composed of 15 amino acids. The novelty is that, despite the wave-like behavior already being demonstrated for large molecules, such a behavior was demonstrated here for fragile biomolecules, "the building blocks of life", at room temperature. The excited comment published on the site of MIT Technology Review was that:

This work paves the way for the study of the quantum properties of biomolecules and sets the scene for experiments that exploit the quantum nature of enzymes, DNA, and perhaps one day simple life forms such as viruses [17].

The two theoretical questions that ignited the debate are how to justify the quantum wavelike properties of macroscopic complex objects, such as fragile biomolecules, and how to justify the quantum nature of biological processes evidently occurring at room temperature. We propose that the proper theoretical framework for an answer to the questions, giving at the same time a measure of semantic information, is provided by QFT [18-20] and specifically by the QFT formalism of the doubling of the degrees of freedom (DDF) for finite temperature [21] and dissipative systems [22].

The "two-slit experiment", to which the just quoted paper on the polypeptide refers to, has been indeed described by using the Wigner function $W(x, p)$ which provides an immediate example of the powerfulness of the DDF formalism. Use of $W(x, p)$ requires in fact the doubling of the canonical set of coordinates $(x, p)$ by introducing $x_{+}=x+y / 2$ and $x_{-}=x-y / 2$, so that the doubled set of coordinates $\left(x_{+}, p_{+}\right)$and $\left(x_{-}, p_{-}\right)$(or $\left(x, p_{x}\right)$ and $\left.\left(y, p_{y}\right)\right)$ describes the system. The result $[9,23]$ is that the diffraction pattern, displaying the quantum wave-like behavior, occurs only when the particle goes forward (in time) through one slit and backward (in time) through the other one, namely $\left|x_{+}(t)-x_{-}(t)\right|>0$. On the contrary, when $x_{+}(t)=x_{-}(t)$, then no diffraction pattern appears on the screen, i.e., the particle behaves classically.

The DDF plays a crucial role in the QFT of dissipative systems and whenever one deals with phase transitions in non-equilibrium systems. The "phases", or space of the states, through which these systems, including biological ones, evolve in time, are physically inequivalent representations of the canonical commutation relations (for bosons, or anticommutation relations for fermions; CCR or CAR, respectively), and therefore unitarily inequivalent representations of the CCR (or CAR) (the QFT foliation of the space of the states). The study of dissipative systems thus cannot be carried on within QM since there all the representation of the CCR (CAR) are unitarily equivalent (as stated by the von Neumann theorem). The proper framework is the one of QFT [9,24], more precisely finite temperature QFT.

This leads us again to DDF, as realized in Thermo Field Dynamics (TFD) formalism [21,25] (see also [9], chs. 5-6; [6]). In TFD it appears clearly that the role played by the DDF is to include in the description the thermal bath, or environment, in which the system is embedded. In this way, the global system (the system under study and its environment) is a closed system and the canonical formalism of QFT can be used. This is a central point in our discussion: the openness of finite temperature (equilibrium) systems, of dissipative systems (finite temperature non-equilibrium systems, including biological systems), and of the semantic information measure all require the inclusion of the environment (the context in CSI and/or the actual world in TCSSI) in the formalism, in order to be formulated within the available canonical formalism. This one is, indeed, devised to account only for closed systems. Without entering technical questions, we only mention that the DDF is realized by adopting the non-commutative Hopf algebra - recalling that the non-commutativity of the algebras is essential for granting logical ordering in topological computer science [10] -, and by introducing the tensor product of the system Hilbert space and the environment Hilbert space. It can be also shown that the equilibrium TFD is equivalent to the $C^{*}$ algebra formalism.

Schematically, we can say that pillars of QFT $[9,21,25]$ are: 
1. The Haag theorem [26] stating the unitary inequivalence between the interacting field representations of the CCR (CAR) and the physical field representations in terms of which observables are described. It implies that, within the resulting non-perturbative formalism, the same basic dynamical interaction may be realized in infinitely many unitarily (i.e., physically) inequivalent representations. Of course, this constitutes a necessary condition for a QFT modelling of non-equilibrium systems evolving over trajectories through different phases (phase transition processes).

2. The use of the Bogoliubov transformations [27] mapping one representation into another unitarily inequivalent one, and thus a boson condensate into another physically inequivalent one. Substantially, this shows the isomorphism of the realizations of the CCR (CAR) in the different representations including their respective topologically non-trivial properties (the corresponding $\mathrm{C}^{*}$ algebras).

3. The spontaneous breakdown of symmetry (SBS), by which the system ground state is not invariant under the full continuous symmetry group of the basic dynamical equations (the Lagrangian). In such a case, the Goldstone theorem [28-30] states the dynamical formation of long-range correlations among the system elementary constituents. The associated boson quanta are called Nambu-Goldstone (NG) quanta. Their condensation in the vacuum (the system ground state) manifests itself in the observable ordered patterns (vacuum structure). For example in ferromagnets, NG are the spin wave quanta or magnons; in crystals, NG bosons are the phonons, quanta of the elastic waves; in polar systems, NG are dipole wave quanta, as e.g. in biological systems where dipole waves span the system [18-20,31]; in superconductors, NG are the Cooper pairs, etc. These NG quanta do not appear in the spectrum of physical excitations in the case that a gauge field (e.g., the electromagnetic field) is present, although they are still responsible of the vacuum structure (the Anderson-Higgs-Kibble mechanism) [9,21]. Different condensates are identified by different values of the "order parameter" (e.g., the magnetization in the ferromagnetic case), which thus acts as a label for the unitarily inequivalent representations corresponding to the different condensates. Therefore, the order parameter identified with the condensation density $N$ turns out to be a dynamic robust memory code in complex systems, biological $[18,32]$ and neural systems before all $[19,20]$.

4. The coherent vacuum structure dynamically generated in the condensation of long-range NG boson modes (therefore as an effect of SBS, see point 3). Coherence is observed to occur in a wide range of temperatures; diamond crystals at atmospheric pressure burn in absence of oxygen at about $3545^{\circ} \mathrm{C}$; in the presence of oxygen at $800^{\circ} \mathrm{C}$; the critical temperature (Curie temperature) for the loss of magnetization of iron is about $770{ }^{\circ} \mathrm{C} ; T_{C}=-252{ }^{\circ} \mathrm{C}$ is the critical temperature for some superconductors. The observed high stability of ordered patterns and their dynamical origin in the SBS phenomenon thus shows that the coherent vacuum structure is protected by the unitarily inequivalence among representations in QFT (in QM the decoherence phenomenon may occur since, due to the von Neumann theorem, all the representations may be related by unitary transformations washing out coherent properties). Moreover, coherence is responsible of the transition from the microscopic behavior of the system components to the macroscopic behavior. This means that the notion of macroscopic quantum system may be thus introduced by considering that characteristic macroscopic properties have their necessary physical justification at the microscopic level of QFT. For example, the order parameter is a classical field since its value is independent of quantum fluctuations. In a coherent state the ratio of quantum fluctuations to the condensate number is indeed proportional to $1 /|\alpha|$, where $|\alpha|$ is the modulus of the coherence strength. A further property of coherence manifest as entanglement among the condensate components $[9,22,33]$.

All of this leads us again to consider the quantum field-particle duality considered above. The observed macroscopic properties of quantum origin are manifestations of "the in-phase behavior" of long-range NG boson condensation. These NG modes enter in the formal mathematical description as "phase modes" and coherence consists in a very much reduced phase uncertainty $\Delta \varphi$, which due to the number-phase uncertainty relation, corresponds to a huge value on the number uncertainty 
$\Delta N$, which is only possible when $N$ is very large (infinite in the limit $\Delta \varphi \rightarrow 0$ ). This brings us back to the need to use fields, which, by definition, are infinite number of degrees of freedom systems. The suggestion is thus that a possible answer to the issues recently addressed in [16] must be searched in the framework of QFT, being out of reach in QM.

\section{Conclusions}

To conclude, the entropy measure associated with the DDF modeling of dissipative quantum systems can be defined as a measure of the system-environment entanglement ([9], pp. 212-215) to be the measure of semantic information we are searching for. See $[6,10]$ for a discussion about this notion and its relationship with the non-commutative deformed Hopf coalgebras. Along the lines there depicted, an information-theoretic approach to quantum computing based on a "doubled" and then "semantic" qubits may be formulated. By the help of Pauli matrices, integer numbers and therefore Krönecker delta (not Dirac delta) may be dealt with [10]. On these matrices, we can implement directly the "quantum Eigenlogic" [34]. That is, a logic of operators where the truth-table semantics is provided directly by the eigenvalue structure of Pauli matrices. As recently demonstrated, this can be directly related to the universality of the quantum gates [34], so to grant that the semantic information according to the DDF approach, satisfies not only in a physical but also in a logical consistent way the TSSI requirement of a "well-formed, meaningful, and truthful" information processing. As a first step, to implement this approach toward the realization of a QFT processor, a group of researchers (at the Labs of the Institute of Microelectronics and Microsystems (IMM) of the Bologna Research Area of the Italian National Research Council (CNR)) has developed a patented electro-optical microchip. This is a fully programmable micro-diffraction grating (PMDG), based on the control over the coherent light waveforms produced by one mini-laser. The control is exerted by electric fields through electrodes on the top of the waveguides (till $10^{3}$ (or actually $10^{2}$ ) guides in 2 $\mathrm{cm}^{2}$ ), hollowed into the electro-optical crystal substrate, and deposited in planar technology on a silicon plate. In this way, it is possible to perform full control over the electromagnetic waveforms, their phase shifts (see [35,36]), and their phase interferences to obtain qubits without the use of statistics of entangled single photon polarization states. Indeed, by using suitable DAC devices, it is possible "to depict" any classical or quantum logical port [34] directly on the dynamic substrate of entangled photon condensates, i.e., of interfering electromagnetic waves.

Author Contributions: The authors contributed equally to the paper.

Funding: This research received no external funding.

Conflicts of Interest: The authors declare no conflict of interest.

\section{References}

1. Shannon, C.E. The Mathematical Theory of Communication; University of Illinois Press: Urbana, IL, USA, 1949.

2. Carnap, R.; Bar-Hillel, Y. An outline of a theory of semantic information. In Languange and Information: Selected Essays on Their Theory and Application; Addison-Wesley: Reading, MA, USA; London, UK, 1964; pp. 221-274.

3. Carnap, R. Meaning and Necessity: A Study in Semantics and Modal Logic; Chicago UP: Chicago, IL, USA, 1956.

4. Rovelli, C. Relational quantum mechanics. Int. J. Theor. Phys. 1996, 35, 1637-1678.

5. Floridi, L. Semantic conceptions of information. The Stanford Encyclopedia of Philosophy (Winter 2019 Edition); Zalta, E.N., Ed.; Available online: https://plato.stanford.edu/archives/win2019/entries/information-semantic/ (accessed on 9 April 2020).

6. Basti, G. The quantum field theory (QFT) dual paradigm in fundamental physics and the semantic information content and measure in cognitive sciences. In Representation of Reality in Humans, other Living Animals, and Machines; Dodig-Crnkovic, R., Giovagnoli, R., Eds.; Springer Int. Publ.: Cham, Switzerland; 2017; pp. 177-210.

7. Kolmogorov, A.N. Foundations of the Theory of Probability, 2nd ed.; Chelsea Publishing: New York, NY, USA, 1956. 
8. Adriaans, P. A critical analysis of Floridi's theory of semantic information. Know. Technol. Policy 2010, 23, 41-56.

9. Blasone, M.; Jizba, P.; Vitiello, G. Quantum Field Theory and its Macroscopic Manifestations. Boson Condensation, Ordered Patternsand Topological Defects; Imperial College Press: London, UK, 2011.

10. Basti, G.; Capolupo, A.; Vitiello, G. Quantum Field Theory and Coalgebraic Logic in Theoretical Computer Science. Prog. Bioph. Mol. Biol. 2017, 130, 39-52.

11. Brentano, F. Psychologie Vom Empirischen Standpunkt; Duncker \& Humblot: Leipzig, Germany, 1874.

12. Damasio, A. The Strange Order of Things. Life, Feeling and the Making of Cultures; Pantheon Books: New York, NY, USA, 2018.

13. Elnitski, L. Epigenetics. NIH-National Humane Genome Research Institute. 2019. Available online: https://www.genome.gov/genetics-glossary/Epigenetics (accessed on 9 April 2020).

14. Barbieri, M. The Organic Codes: An Introduction to Semantic Biology; Cambridge UP: Cambridge, UK, 2003.

15. Barbieri, M. Biosemiotics: A new understanding of life. Naturwissenschaften 2008, 95, 577-599.

16. Shayeghi, A.; Rieser, P.A.; Richter, G.; Sezer, U.; Rodewald, J.H.; Geyer, P.; Martinez, T.J.; Arndt, M. Matterwave interference of a native polypeptide. Nat. Commun. 2020, 11, 1447.

17. A Natural Biomolecule has been Measured Acting Like a Quantum Wave for the First Time. MIT Technology Review 9 November 2019. Available online: https://www.technologyreview.com/2019/11/09/238365/a-natural-biomolecule-has-been-measured-acting-in-a-quantum-wavefor-the-first-time/ (accessed on 19 April 2020).

18. Del Giudice, E.; Doglia, S.; Milani, M.; Vitiello, G. A quantum field theoretical approach to the collective behavior of biological systems. Nucl. Phys. B 1985, 251, 375.

19. Freeman, W.J.; Vitiello, G. Nonlinear brain dynamics as macroscopic manifestation of underlying many-body field dynamics. Phys. Life Rev. 2006, 3, 93-118.

20. Freeman, W.J.; Vitiello, G. Dissipation and spontaneous symmetry breaking in brain dynamics. J. Phys. A Math. Theor. 2008, 41, 304042.

21. Umezawa, H. Advanced Field Theory: Micro, Macro and Thermal Concepts; American Institute of Physics: New York, NY, USA, 1993.

22. Vitiello, G. Links. Relating different physical systems through the common QFT algebraic structure. Lect. Notes Phys. 2017, 718, 165-205.

23. Blasone, M.; Srivastava, Y.N.; Vitiello, G.; Widom, A. Phase coherence in quantum Brownian motion. Annals Phys. 1998, 267, 61-74.

24. Celeghini, E.; Rasetti, M.; Vitiello, G. Quantum dissipation. Annals Phys. 1992, 215, 156-170.

25. Umezawa, H. Development of concepts in quantum field theory in half century. Math. Jpn. 1995, 41, 109124.

26. Haag, R. On quantum field theories. Matemat.-Fys. Meddel. 1955, 29, 1-37.

27. Bogoliubov, N.N. On a new method in the theory of superconductivity. Nuovo Cimento 1958, 7, 794-805.

28. Goldstone, J. Field Theories with Superconductor Solutions. Nuovo Cimento 1961, 19, 154-164.

29. Goldstone, J.; Salam, A.; Weinberg, S. Broken Symmetries. Phys. Rev. 1962, 127, 965-970.

30. Nambu, Y.; Jona-Lasinio, G. Dynamical Model of Elementary Particles Based on an Analogy with Superconductivity. Phys. Rev. 1961, 122, 345-358.

31. Fröhlich, H. Long range coherence and energy storage in biological systems. Int. J. Q. Chem. 1968, II, 641649.

32. Del Giudice, E.; Pulselli, R.; Tiezzi, E. Thermodynamics of irreversible processes and quantum field theory: An interplay for understanding of ecosystem dynamics. Ecol. Modell. 2009, 220, 1874-1879.

33. Sabbadini, S.A.; Vitiello, G. Entanglement and phase-mediated correlations in quantum field theory. Application to brain-mind states. App. Sci. 2019, 9, 3203.

34. Toffano, Z.; Dubois, F. Adapting logic to physics: The quantum-like eigenlogic program. Entropy 2020, 22, 139. 
35. Basti, G.; Bentini, G.G.; Chiarini, M.; Parini, A.; Artoni, A.; Braglia, F.; Braglia, S.; Farabegoli, S. Sensor for security and safety applications based on a fully integrated monolithic electro-optical programmable microdiffractive device. In Proceedings SPIE 11159, Electro-Optical and Infrared Systems: Technology and Applications XVI, 1115907; SPIE Publ.: Strasbourg, France, 2019; pp. 1-12.

36. Parini, A.; Chiarini, M.; Basti, G.; Bentini, G.G. Lithium niobate-based programmable micro-diffraction device for wavelength-selective switching applications. In Proceedings SPIE 11163, Emerging Imaging and Sensing Technologies for Security and Defence IV, 111630C; SPIE Publ.: Strasbourg, France, 2019; pp. 1-7.

(C) 2020 by the authors. Licensee MDPI, Basel, Switzerland. This article is an open access article distributed under the terms and conditions of the Creative Commons Attribution (CC BY) license (http://creativecommons.org/licenses/by/4.0/). 